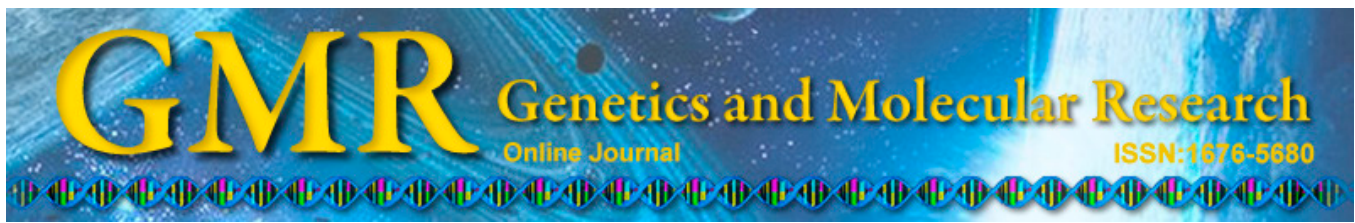

\title{
Matrix metalloproteinase variants associated with risk and clinical outcome of esophageal cancer
}

\author{
L. Zhang, R.-X. Xi and X.-Z. Zhang \\ Department of Radiation Oncology, \\ The First Affiliated Hospital of Xi'an Jiaotong University, Xi'an, Shanxi, China \\ Corresponding author: X.-Z. Zhang \\ E-mail: zhangxz9149@163.com
}

Genet. Mol. Res. 14 (2): 4616-4624 (2015)

Received July 17, 2014

Accepted October 29, 2014

Published May 4, 2015

DOI http://dx.doi.org/10.4238/2015.May.4.20

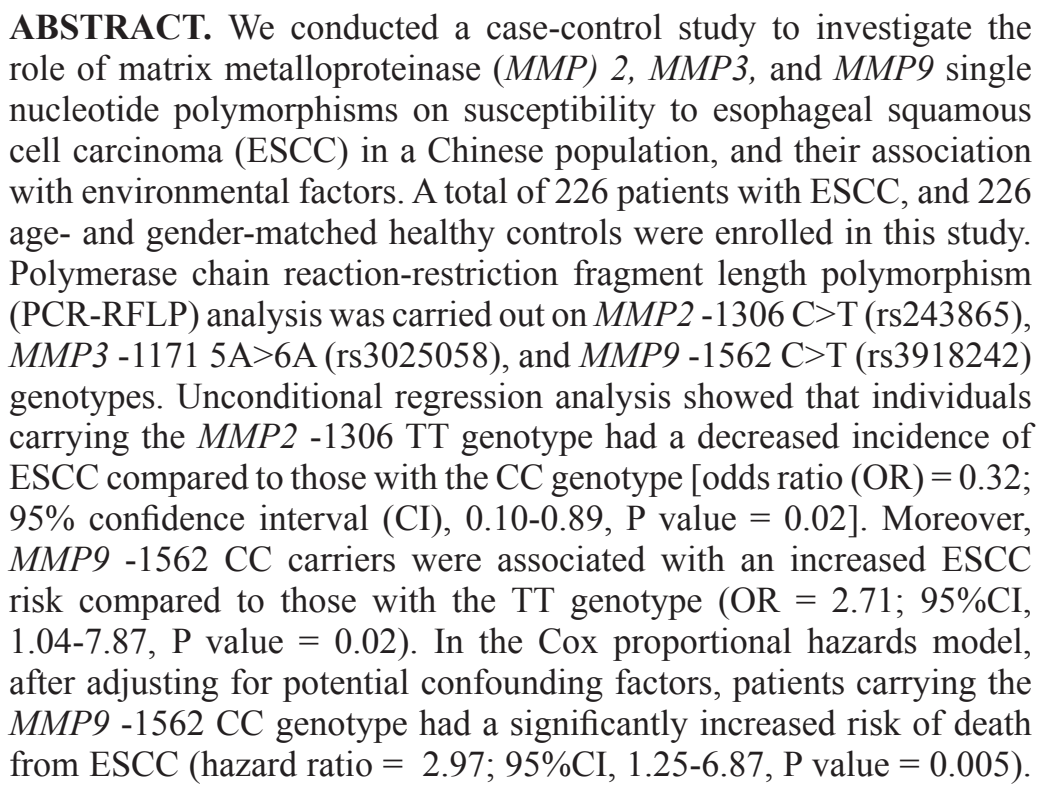


In conclusion, this study showed that the MMP2 -1306 TT and MMP9 -1562 CC genotypes were associated with increased ESCC, and patients carrying the $M M P 9-1562 \mathrm{CC}$ genotype had a significantly increased risk of death from ESCC.

Key words: Esophageal squamous cell carcinoma; Polymorphism; Matrix metalloproteinases

\section{INTRODUCTION}

Esophageal squamous cell carcinoma (ESCC), which occurs at a very high frequency in China, is one of the most common fatal cancers worldwide and the number of deaths is increasing (Thallinger et al., 2012; Shang and Wang, 2013; Lin et al., 2013). Surgical resection is a standard treatment for ESCC patients but its use depends on the stage and development of the tumor. While multi-therapeutic strategies such as chemo- and radiotherapy are employed to tackle ESCC, the overall 5-year survival rate remains low at 10-30\% (Stoner et al., 2007; Nakajima and Kato, 2013). In addition, tumor resistance to radio- and chemotherapy has also been reported (Xie et al., 2009).

Therefore, there is an urgent need to find new therapeutic methods to improve the clinical treatment of ESCC patients. It has been demonstrated that some pathogenic factors such as cancer-related genes are associated with the progression and development of ESCC. The matrix metalloproteinases (MMPs) are a family of zinc-dependent proteolytic enzymes capable of degrading the extracellular matrix. MMPs play a key role in the physiological degradation of the extracellular matrix in angiogenesis, tissue repair, and tissue morphogenesis (Freije et al., 2003). They also regulate cell growth and inflammation by cleaving non-matrix proteins such as growth factors, cytokines and chemokines, and their respective receptors. Previous studies show that MMPs play a critical role in the invasion and metastasis of most malignancies (Stetler-Stevenson et al., 1993; Nagase and Woessner, 1999; Stetler-Stevenson and $\mathrm{Yu}, 2001$; Freije et al., 2003). MMP2, MMP3, and MMP9 are three important members of the MMP family, and previous studies reported that polymorphisms of MMP2, MMP3, and MMP9 were associated with the risk and prognosis of several cancers such as bladder, gastric, breast, and oral cancer, as well as osteosarcoma (Pereira et al., 2012; Saeed et al., 2013; Yang et al., 2014; Wieczorek et al., 2014; Yan et al., 2014; Wen et al., 2014). Furthermore, there have been several reports on the association between MMP2, MMP3, and MMP9 polymorphisms, and the development and prognosis of ESCC, but the results are inconsistent (Zhang et al., 2005; Wu et al., 2008; Bradbury et al., 2009; Peng et al., 2010; Li et al., 2013). The discrepancy between these results may be caused by variable genetic distributions in different populations, or dissimilar study designs and sample sizes.

Therefore, we conducted a case-control study to investigate the role of MMP2, MMP3, and MMP9 single nucleotide polymorphisms (SNPs) on susceptibility to ESCC in a Chinese population, and their association with environmental factors.

\section{MATERIAL AND METHODS}

\section{Population}

A hospital-based case-control study was conducted. A total of 226 ESCC patients 
and 226 age- and gender-matched healthy controls from the First Affiliated Hospital of Xi'an Jiaotong University were enrolled into our study, between January 2008 and December 2010. ESCC patients were newly diagnosed and histopathologically confirmed independently by two pathologists. Patients who had secondary or recurrent tumors or a history of other malignant neoplasms, and inadequate organ function were excluded from the case group.

Age- and sex-matched controls $(\mathrm{N}=226)$ were selected from subjects who came to our hospital for a health check-up. Controls who had a history of cancer were excluded. Informed consent was obtained from all cases and control subjects when they agreed to participate in our study. The study protocol was approved by the ethics committee of the First Affiliated Hospital of Xi'an Jiaotong University.

Demographic and related lifestyle factors such as gender, age, dietary habits, alcohol consumption, tobacco smoking, and family history of cancer were collected by a face-to-face questionnaire. These were completed by trained interviewers who were not aware of the study hypothesis.

Smoking status was divided into non-smokers and smokers. The latter were defined as those who smoked more than one cigarette/pipe per day for at least $50 \%$ of the year. Alcohol drinking status was divided into non-drinkers, and drinkers who consumed $50 \mathrm{~g}$ alcohol (200 $\mathrm{mL}$ beer, $100 \mathrm{~mL}$ wine, and $50 \mathrm{~mL}$ spirits) per week for at least $50 \%$ of the year. Family history referred to first- or second-degree relatives such as parents, grandparents, siblings, and offspring.

All patients were followed up until December 30,2012, with a median follow-up time of 35.6 months (range, 2-60 months). All patients were followed up by telephone every four weeks until death or the end of study. Overall survival (OS) was calculated from the date of enrolling in this study to the date of death or last clinical follow-up.

\section{Genotyping}

A 5-mL venous blood sample was collected from each subject following interview. The blood samples were kept at $-20^{\circ} \mathrm{C}$ until needed, with $0.5 \mathrm{mg} / \mathrm{mL}$ ethylenediaminetetraacetic acid used as an anticoagulant. Genomic DNA was extracted from whole blood using a TIANamp blood DNA kit (Tiangen Biotech; Beijing, China) according to manufacturer instructions.

Polymerase chain reaction-restriction fragment length polymorphism (PCR-RFLP) analysis was carried out on $M M P 2-1306 \mathrm{C}>\mathrm{T}$ (rs243865), MMP3 -1171 5A >6A (rs3025058), and $M M P 9-1562 \mathrm{C}>\mathrm{T}$ (rs3918242) genotypes. PCR primers for $M M P 2-1306 \mathrm{C}>\mathrm{T}$ (rs243865), $M M P 3-1171$ 5A >6A (rs3025058), and $M M P 9-1562 \mathrm{C}>\mathrm{T}(\mathrm{rs} 3918242)$ were designed using the Primer 5.0 software according to the manufacturer instructions. Primer pairs for MMP2 -1306 C $>$ T (rs243865) were 5'-CTGACCCCCAGTCCTATCTGCC-3' and 5'-TGTTGGGAACGC CTGACTTCAG-3'; for MMP3 -1171 5A>6A (rs3025058) were 5' ACGTTGGATGGTCCTC ATATCAATGTGGCC-3' and 5'-ACGTTGGATGCTATGGTTCTCCATTCCTTTG-3'; and for MMP9 -1562 C>T (rs3918242) were 5'-GCCTGGCACATAGTAGGCCC-3' and 5'-TTCCTAGCCAGCCGGCATC-3'.

PCR amplification conditions were as follows: an initial denaturation for $5 \mathrm{~min}$ at $95^{\circ} \mathrm{C}$; followed by $35 \mathrm{step}$ cycles of denaturation at $95^{\circ} \mathrm{C}$ for $30 \mathrm{~s}$, annealing at $62^{\circ} \mathrm{C}$ for $30 \mathrm{~s}$, and extension at $72^{\circ} \mathrm{C}$ for $30 \mathrm{~s}$; followed by a final extension at $72^{\circ} \mathrm{C}$ for $10 \mathrm{~min}$. PCR products were verified on a $2 \%$ agarose gel stained with ethidium bromide and observed under ultraviolet light. The results were confirmed by sequencing the PCR products using an automated sequencing system. For quality control, $10 \%$ of the cases and controls were randomly selected to be genotyped again by different investigators and $100 \%$ reproducibility was obtained. 


\section{Statistical analysis}

All analyses were performed with the SPSS Version 16.0 software (SPSS Inc., Chicago, IL, USA). Continuous variables are reported as means \pm standard deviation (SD), while categorical variables are shown as frequencies and percentages. Demographic and clinical variables were compared across genotypes using the chi-square test. The association between genetic polymorphisms and the risk of ESCC was estimated by a conditional multiple logistical regression model, and the results are expressed as odds ratios (ORs) and 95\% confidence intervals $(95 \% \mathrm{CIs})$. The OR $(95 \% \mathrm{CI})$ was adjusted for potential risk factors such as gender, age, smoking and drinking status, and family history of cancer. The association between MMP7 and MMP9 mRNA and overall survival of ESCC was estimated using Cox's proportional hazard model; the most frequent genotype was used as the reference group. Statistical significance was defined as a two-sided $\mathrm{P}$ value $<0.05$.

\section{RESULTS}

The characteristics of the ESCC cancer patients and controls are shown in Table 1. There was no significant difference between the cases and controls in terms of gender and age. When ESCC patients and healthy controls were compared in terms of demographic characteristics, ESCC patients were more likely to be older, have a smoking and drinking habit, and have a family history of cancer $(\mathrm{P}<0.05$; Table 1$)$.

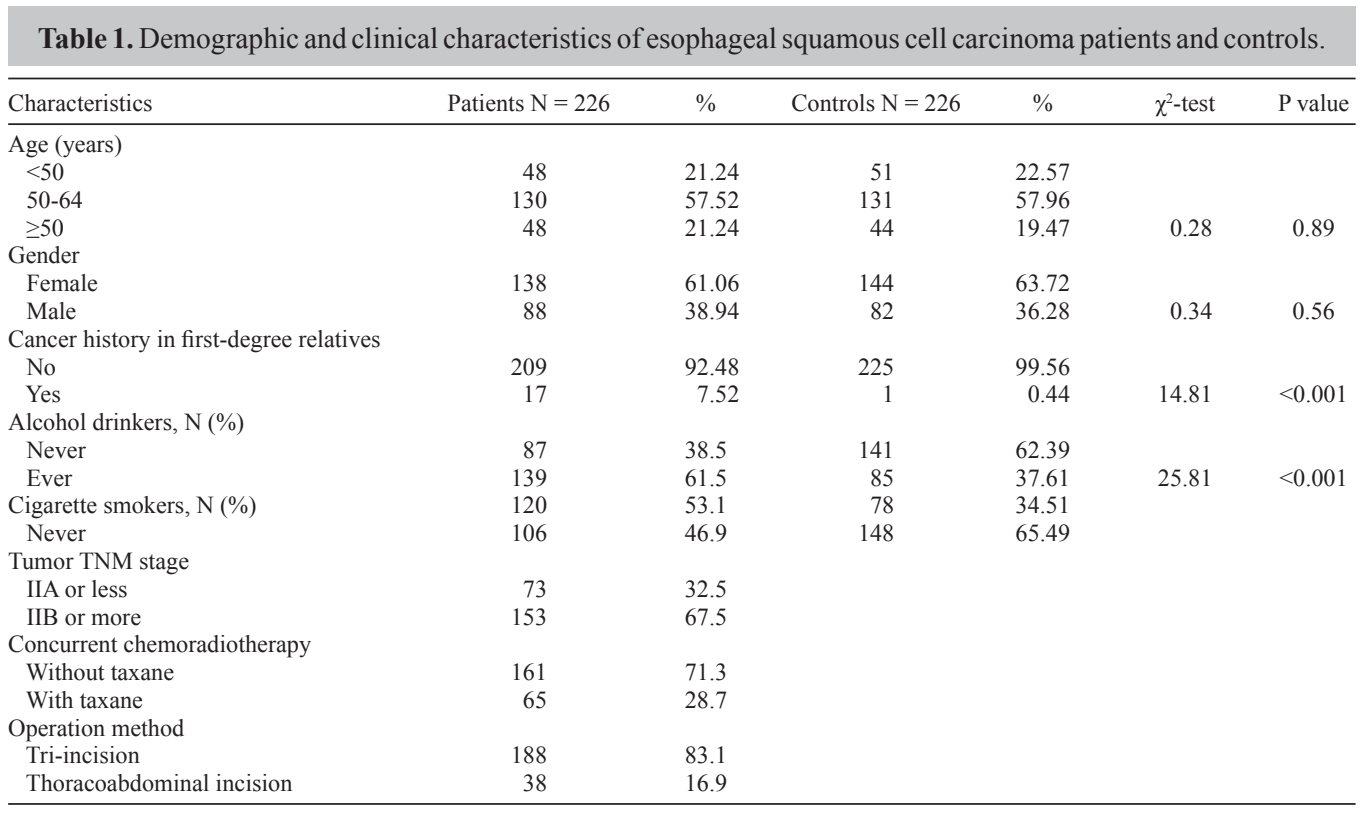

In our study population, the genotype and allele distributions of $M M P 2-1306 \mathrm{C}>\mathrm{T}$, $M M P 3-11715 \mathrm{~A}>6 \mathrm{~A}$, and $M M P 9-1562 \mathrm{C}>\mathrm{T}$ in the controls were in line with Hardy-Weinberg equilibrium. The genotype and allele distributions of the three SNPs in the ESCC cases and control subjects are shown in Table 2. Unconditional regression analysis showed that individu- 
als carrying the MMP2 -1306 TT genotype had a decreased incidence of ESCC compared to those with the $\mathrm{CC}$ genotype $(\mathrm{OR}=0.32 ; 95 \% \mathrm{CI}, 0.10-0.89, \mathrm{P}$ value $=0.02)$. Moreover, $M M P 9$ $-1562 \mathrm{CC}$ carriers were associated with an increased ESCC risk compared to those with the TT genotype $(\mathrm{OR}=2.71 ; 95 \% \mathrm{CI}, 1.04-7.87, \mathrm{P}$ value $=0.02)$.

Table 2. Genotype distributions of $M M P 2-1306 \mathrm{C}>\mathrm{T}, M M P 3-11715 \mathrm{~A}>6 \mathrm{~A}$, and $M M P 9-1562 \mathrm{C}>\mathrm{T}$ in esophageal squamous cell carcinoma patients and controls.

\begin{tabular}{|c|c|c|c|c|c|c|}
\hline Gene & Patients $\mathrm{N}=226$ & $\%$ & Controls $\mathrm{N}=226$ & $\%$ & OR $(95 \% \mathrm{CI})^{1}$ & $P$ value \\
\hline \multicolumn{7}{|c|}{$M M P 2-1306 \mathrm{C}>\mathrm{T}$} \\
\hline $\mathrm{CC}$ & 136 & 60.18 & 136 & 60.3 & 1.0 (Ref.) & - \\
\hline CT & 73 & 32.30 & 73 & 32.4 & $0.88(0.58-1.34)$ & 0.54 \\
\hline TT & 17 & 7.52 & 17 & 7.3 & $0.32(0.10-0.89)$ & 0.02 \\
\hline \multicolumn{7}{|c|}{$M M P 3-11715 \mathrm{~A}>6 \mathrm{~A}$} \\
\hline $5 \mathrm{~A} / 5 \mathrm{~A}$ & 163 & 72.3 & 168 & 74.5 & 1.0 (Ref.) & - \\
\hline $5 \mathrm{~A} / 6 \mathrm{~A}$ & 37 & 16.4 & 35 & 15.3 & $1.09(0.63-1.88)$ & 0.74 \\
\hline $6 \mathrm{~A} / 6 \mathrm{~A}$ & 26 & 11.3 & 23 & 10.2 & $1.17(0.61-2.23)$ & 0.62 \\
\hline \multicolumn{7}{|c|}{$M M P 9-1562 \mathrm{C}>\mathrm{T}$} \\
\hline TT & 164 & 72.5 & 173 & 76.4 & 1.0 (Ref.) & - \\
\hline CT & 44 & 19.4 & 46 & 20.5 & $1.01(0.62-1.65)$ & 0.96 \\
\hline $\mathrm{CC}$ & 18 & 8.1 & 7 & 3.1 & $2.71(1.04-7.87)$ & 0.02 \\
\hline
\end{tabular}

${ }^{1}$ Adjusted for gender, age, alcohol drinking, and cigarette smoking. OR, odds ratio; $95 \% \mathrm{CI}, 95 \%$ confidence interval.

We analyzed the effect of $M M P 2-1306 \mathrm{C}>\mathrm{T}, M M P 3-11715 \mathrm{~A}>6 \mathrm{~A}$, and $M M P 9$ $-1562 \mathrm{C}>\mathrm{T}$ on the overall survival of ESCC patients (Table 3). During the follow-up period, 74 patients $(32.74 \%)$ died. In the Cox proportional hazards model, after adjusting for potential confounding factors, patients carrying the $M M P 9-1562 \mathrm{CC}$ genotype had a significantly increased risk of death from ESCC (hazard ratio $=2.97 ; 95 \% \mathrm{CI} \%, 1.25-6.87$, $\mathrm{P}$ value $=0.005$, Figure 1). However, we observed no significant association between the $M M P 2-1306 \mathrm{C}>\mathrm{T}$ and $M M P 3-11715 \mathrm{~A}>6 \mathrm{~A}$ polymorphisms, and overall survival of ESCC patients.

Table 3. Effect of $M M P 2-1306 \mathrm{C}>\mathrm{T}, M M P 3-11715 \mathrm{~A}>6 \mathrm{~A}$, and $M M P 9-1562 \mathrm{C}>\mathrm{T}$ on the overall survival of esophageal squamous cell carcinoma (ESCC) patients.

\begin{tabular}{|c|c|c|c|c|c|c|c|}
\hline SNPs & ESCC & $\%$ & Death $N=74$ & $\%$ & 5 -year survival rate $(\%)$ & $\mathrm{HR}(95 \% \mathrm{CI})$ & $P$ value \\
\hline \multicolumn{8}{|c|}{$M M P 2-1306 \mathrm{C}>\mathrm{T}$} \\
\hline $\mathrm{CC}$ & 136 & 60.3 & 42 & 56.4 & 69.12 & 1.0 (Ref.) & - \\
\hline CT & 73 & 32.4 & 25 & 34.5 & 64.38 & $1.15(0.63-2.10)$ & 0.62 \\
\hline TT & 17 & 7.3 & 7 & 9.1 & 58.82 & $1.33(0.44-3.67)$ & 0.55 \\
\hline \multicolumn{8}{|c|}{$M M P 3-11715 \mathrm{~A}>6 \mathrm{~A}$} \\
\hline $5 \mathrm{~A} / 5 \mathrm{~A}$ & 163 & 72.3 & 52 & 70.3 & 68.10 & 1.0 (Ref.) & \\
\hline $5 \mathrm{~A} / 6 \mathrm{~A}$ & 37 & 16.4 & 13 & 16.9 & 64.86 & $1.10(0.50-2.32)$ & 0.79 \\
\hline $6 \mathrm{~A} / 6 \mathrm{~A}$ & 26 & 11.3 & 9 & 12.8 & 65.38 & $1.09(0.42-2.58)$ & 0.85 \\
\hline \multicolumn{8}{|c|}{$M M P 9-1562 \mathrm{C}>\mathrm{T}$} \\
\hline TT & 164 & 72.5 & 43 & 58.7 & 73.78 & 1.0 (Ref.) & - \\
\hline CT & 44 & 19.4 & 17 & 22.6 & 61.36 & $1.47(0.72-2.94)$ & 0.24 \\
\hline $\mathrm{CC}$ & 18 & 8.1 & 14 & 18.7 & 22.22 & $2.97(1.25-6.87)$ & 0.005 \\
\hline
\end{tabular}

HR, hazard ratio; 95\%CI, 95\% confidence interval. 


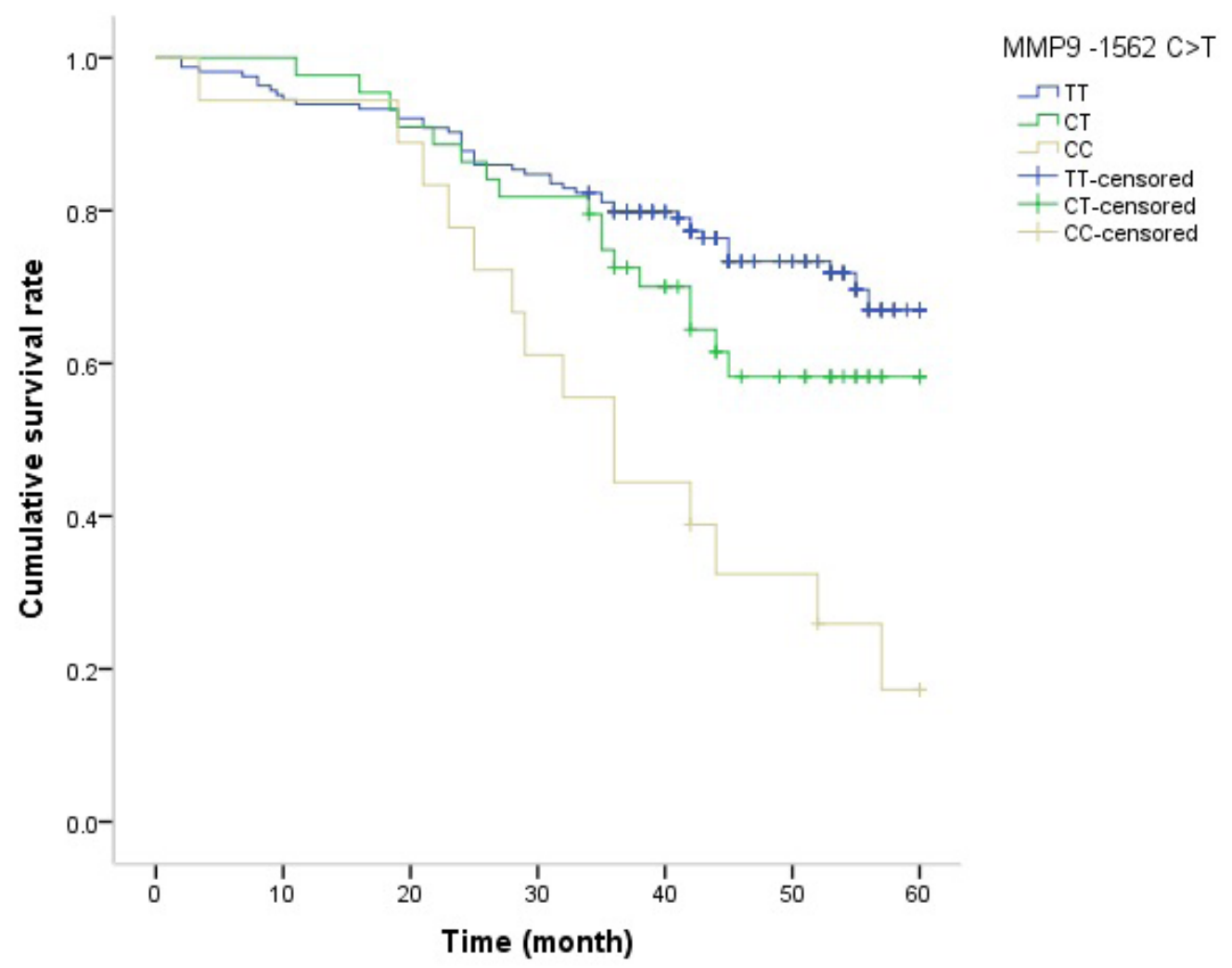

Figure 1. Kaplan-Meier analysis of overall survival for $M M P 9-1562 \mathrm{C}>\mathrm{T}$.

\section{DISCUSSION}

Identification of the genes involved in the genetic predisposition or progression of cancer has an important role in clinical practice and basic medicine research. In this hospitalbased case-control study of ESCC, we investigated the association of $M M P 2-1306 \mathrm{C}>\mathrm{T}$, $M M P 3-11715 \mathrm{~A}>6 \mathrm{~A}$, and $M M P 9-1562 \mathrm{C}>\mathrm{T}$ polymorphisms with the development and prognosis of ESCC in a Chinese population. Our multivariate logistic analysis showed that the $M M P 2-1306$ TT and MMP9 -1562 CC genotypes were associated with increased ESCC, and patients carrying the $M M P 9-1562 \mathrm{CC}$ genotype had a significantly increased risk of death from ESCC.

Our study found that the MMP2 $-1306 \mathrm{C}>\mathrm{T}$ polymorphism is associated with the development of ESCC. It is well known that MMP2 $-1306 \mathrm{C}>\mathrm{T}$ contains a $\mathrm{C}>\mathrm{T}$ transition at the -1306 position upstream of the transcriptional site, and this polymorphism can abolish the SpI-binding site and downregulate transcriptional activity. Previous studies reported that the $M M P 2-1306 \mathrm{~T}$ allele was associated with lower expression of the $M M P 2$ gene compared with the $\mathrm{C}$ allele (Price et al., 2001). A previous meta-analysis indicated that the MMP2 -1306 CT and TT genotypes were associated with a decreased risk of ESCC compared with the CC genotype (Zhang et al., 2013). Furthermore, several studies reported that overexpression of 
$M M P 2$ was associated with the development and aggressiveness of several cancers such as gastric, bladder, lung, and head and neck cancers (Zhang et al., 2013; Hu et al., 2013; Yan et al., 2014; Yang et al., 2014). Three studies reported the association between the MMP2 -1306 $\mathrm{C}>\mathrm{T}$ polymorphism and risk of ESCC (Lin et al., 2004; O-Charoenrat and Khantapura, 2006; Zhou et al., 2007). Lin et al. (2004) conducted a study in Taiwan, and showed that subjects carrying the CT or TT genotypes had a decreased risk of developing ESCC compared with those having the CC genotype. O-Charoenrat and Khantapura (2006) reported that MMP2 -1306 CT and TT genotypes were associated with a decreased risk of ESCC, and were also correlated with adverse clinicopathological variables. Our findings confirmed those of previous studies (Lin et al., 2004; Zhou et al., 2007).

The MMP9 -1562 C>T polymorphism is located 1562 bp upstream of the transcriptional start site and contains either $\mathrm{C}$ or $\mathrm{T}$, and this allele variation could influence the transcriptional activity of the $M M P 9$ gene. A previous study conducted transient transfections and DNA-protein interaction assays, and found that the T allele of MMP9 -1562 $\mathrm{C}>\mathrm{T}$ had higher promoter activity compared with the $\mathrm{C}$ allele due to the binding of a transcriptional repressor (Zhang et al., 1999). Several previous studies found that the $M M P 9-1562 \mathrm{C}>\mathrm{T}$ polymorphism was associated with an increased risk of lung and bladder cancer and ESCC (Vairaktaris et al., 2008; Lei et al., 2009; Wieczorek et al., 2013). Lei et al. (2009) reported a meta-analysis of eight case-control studies, and discovered that the $M M P 9-1562 \mathrm{C}>\mathrm{T}$ polymorphism was associated with lung cancer risk. Wieczorek et al. (2013) reported that the MMP9-1562 T allele was linked with increased bladder cancer risk. One study reported that the MMP9-1562 C>T polymorphism was associated with increased risk of ESCC, and conversely, another report demonstrated no association (Zhou et al., 2007). The discrepancy between these results may be explained by differences in ethnicities, sources of control subjects, sample size, and also chance. Further studies are needed to confirm the association between the MMP9 $-1562 \mathrm{C}>\mathrm{T}$ polymorphism and ESCC risk.

In our study, we found that the MMP9 $-1562 \mathrm{C}>\mathrm{T}$ polymorphism is correlated with ESCC prognosis. Previous studies also reported that the $-634 \mathrm{G} / \mathrm{C}$ polymorphism was associated with the clinical outcome of colorectal cancer and non-small cell lung cancer (Rollin et al., 2007; Langers et al., 2012). One previous meta-analysis found that overexpression of $M M P-9$ is associated with a poor prognosis for ESCC (Zeng et al., 2013). Further studies are required to confirm this association.

There are several limitations in our study. First, cases and controls were selected from one hospital. Therefore, there is a risk of selection bias because the cases and controls were not a random sample of the ESCC population, and may not accurately represent all patients with ESCC. Second, due to the rarity of ESCC, only a small number of cases were selected. The relatively small sample size could limit the statistical power to find differences between the groups. Third, other genetic factors may affect the development and prognosis of ESCC, and $M M P$ genes may interact with them. Therefore, further studies with large samples are needed to confirm the association between $M M P$ polymorphisms and the development and prognosis of ESCC.

In summary, this study showed that the $M M P 2-1306$ TT and $M M P 9-1562 \mathrm{CC}$ genotypes were associated with an increased risk of ESCC, and patients carrying the MMP9-1562 CC genotype had a significantly increased risk of death from ESCC. This suggests that $M M P$ polymorphisms are predictive markers of risk and clinical outcome for ESCC in the Chinese population. 


\section{REFERENCES}

Bradbury PA, Zhai R, Hopkins J, Kulke MH, et al. (2009). Matrix metalloproteinase 1, 3 and 12 polymorphisms and esophageal adenocarcinoma risk and prognosis. Carcinogenesis 30: 793-798.

Freije JM, Balbín M, Pendás AM, Sánchez LM, et al. (2003). Matrix metalloproteinases and tumor progression. Adv. Exp. Med. Biol. 532: 91-107.

$\mathrm{Hu}$ C, Wang J, Xu Y, Li X, et al. (2013). Current evidence on the relationship between five polymorphisms in the matrix metalloproteinases (MMP) gene and lung cancer risk: a meta-analysis. Gene 517: 65-71.

Langers AM, Verspaget HW, Hawinkels LJ, Kubben FJ, et al. (2012). MMP-2 and MMP-9 in normal mucosa are independently associated with outcome of colorectal cancer patients. Br J. Cancer 106: 1495-1498.

Lei Z, Liu R, Chen J, Zhou Q, et al. (2009). Meta analysis of association between polymorphisms in promoter region of MMPs gene and risk of lung cancer. Zhongguo Fei Ai Za Zhi. 12: 381-386.

Li X, Qu L, Zhong Y, Zhao Y, et al. (2013). Association between promoters polymorphisms of matrix metalloproteinases and risk of digestive cancers: a meta-analysis. J. Cancer. Res. Clin. Oncol. 139: 1433-1447.

Lin SC, Lo SS, Liu CJ, Chung MY, et al. (2004). Functional genotype in matrix metalloproteinases-2 promoter is a risk factor for oral carcinogenesis. J. Oral Pathol. Med. 33: 405-409.

Lin Y, Totsuka Y, He Y, Kikuchi S, et al. (2013). Epidemiology of esophageal cancer in Japan and China. J. Epidemiol. 23: $233-242$.

Nagase H and Woessner JF (1999). Matrix metalloproteinases. J. Biol. Chem. 274: 21491-21494.

Nakajima M and Kato H (2013). Treatment options for esophageal squamous cell carcinoma. Expert Opin. Pharmacother. 14: $1345-1354$.

O-Charoenrat P and Khantapura P (2006). The role of genetic polymorphisms in the promoters of the matrix metalloproteinase-2 and tissue inhibitor of metalloproteinase-2 genes in head and neck cancer. Oral Oncol. 42: 257-267.

Peng B, Cao L, Ma X, Wang W, et al. (2010). Meta-analysis of association between matrix metalloproteinases 2, 7 and 9 promoter polymorphisms and cancer risk. Mutagenesis 25: 371-379.

Pereira AC, Dias do Carmo E, Dias da Silva MA and Blumer Rosa LE (2012). Matrix metalloproteinase gene polymorphisms and oral cancer. J. Clin. Exp. Dent. 4: e297-e301.

Price SJ, Greaves DR and Watkins H (2001). Identification of novel, functional genetic variants in the human matrix metalloproteinase-2 gene: role of $\mathrm{Sp} 1$ in allele-specific transcriptional regulation. J. Biol. Chem. 276: 7549-7558.

Rollin J, Régina S, Vourc'h P, Iochmann S, et al. (2007). Influence of MMP-2 and MMP-9 promoter polymorphisms on gene expression and clinical outcome of non-small cell lung cancer. Lung Cancer 56: 273-280.

Saeed HM, Alanazi MS, Alshahrani O, Parine NR, et al. (2013). Matrix metalloproteinase-2 C(-1306)T promoter polymorphism and breast cancer risk in the Saudi population. Acta Biochim. Pol. 60: 405-409.

Shang L and Wang M (2013). Molecular alterations and clinical relevance in esophageal squamous cell carcinoma. Front Med. 7: 401-410.

Stetler-Stevenson WG and Yu AE (2001). Proteases in invasion: matrix metalloproteinases. Semin Cancer Biol. 11: 143152.

Stetler-Stevenson WG, Liotta LA and Kleiner DE Jr (1993). Extracellular matrix 6: role of matrix metalloproteinases in tumor invasion and metastasis. FASEB J. 7: 1434-1441.

Stoner GD, Wang LS and Chen T (2007). Chemoprevention of esophageal squamous cell carcinoma. Toxicol Appl Pharmacol. 224: 337-349.

Thallinger CM, Kiesewetter B, Raderer M and Hejna M (2012). Pre- and postoperative treatment modalities for esophageal squamous cell carcinoma. Anticancer Res. 32: 4609-4627.

Vairaktaris E, Vassiliou S, Nkenke E, Serefoglou Z, et al. (2008). A metalloproteinase-9 polymorphism which affects its expression is associated with increased risk for oral squamous cell carcinoma. Eur. J. Surg. Oncol. 34: 450-455.

Wen X, Liu H, Yu K and Liu Y (2014). Matrix metalloproteinase 2 expression and survival of patients with osteosarcoma: a meta-analysis. Tumour Biol. 35: 845-848.

Wieczorek E, Reszka E, Jablonowski Z, Jablonska E, et al. (2013). Genetic polymorphisms in matrix metalloproteinases (MMPs) and tissue inhibitors of MPs (TIMPs), and bladder cancer susceptibility. BJU Int. 112: 1207-1214.

Wieczorek E, Wasowicz W, Gromadzinska J and Reszka E (2014). Functional polymorphisms in the matrix metalloproteinase genes and their association with bladder cancer risk and recurrence: a mini-review. Int. J. Urol. 21: 744-752.

Wu J, Zhang L, Luo H, Zhu Z, et al. (2008). Association of matrix metalloproteinases-9 gene polymorphisms with genetic susceptibility to esophageal squamous cell carcinoma. DNA Cell Biol. 27: 553-557. 
Xie L, Song X, Yu J, Wei L, et al. (2009). Fractionated irradiation induced radio-resistant esophageal cancer EC109 cells seem to be more sensitive to chemotherapeutic drugs. J. Exp. Clin. Cancer Res. 28: 68.

Yan Y, Liang H, Li T, Li M, et al. (2014). The MMP-1, MMP-2, and MMP-9 gene polymorphisms and susceptibility to bladder cancer: a meta-analysis. Tumour Biol. 35: 3047-3052.

Yang TF, Guo L and Wang Q (2014). Meta-analysis of associations between four polymorphisms in the matrix metalloproteinases gene and gastric cancer risk. Asian Pac. J. Cancer Prev. 15: 1263-1267.

Zeng R, Duan L, Kong Y, Liang Y, et al. (2013). Clinicopathological and prognostic role of MMP-9 in esophageal squamous cell carcinoma: a meta-analysis. Chin. J. Cancer Res. 25: 637-645.

Zhang B, Henney A, Eriksson P, Hamsten A, et al. (1999). Genetic variation at the matrix metalloproteinase-9 locus on chromosome 20q12.2-13.1. Hum. Genet. 105: 418-423.

Zhang C, Li C, Zhu M, Zhang Q, et al. (2013). Meta-analysis of MMP2, MMP3, and MMP9 promoter polymorphisms and head and neck cancer risk. PLoS One 8: e62023.

Zhang J, Jin X, Fang S, Wang R, et al. (2005). The functional polymorphism in the matrix metalloproteinase-7 promoter increases susceptibility to esophageal squamous cell carcinoma, gastric cardiac adenocarcinoma and non-small cell lung carcinoma. Carcinogenesis 26: 1748-1753.

Zhou G, Zhai Y, Cui Y, Qiu W, et al. (2007). Functional polymorphisms and haplotypes in the promoter of the MMP2 gene are associated with risk of nasopharyngeal carcinoma. Hum. Mutat. 28: 1091-1097. 